\title{
Communication
}

\section{Deficiency of MMP-10 aggravates the diseased phenotype of aged dystrophic mice}

Arantxa Baraibar-Churio1,2,†, Míriam Bobadilla1,2,†, Neira Sáinz1,2,3, Florencio JD Machado2,4, Josune Orbe2,4, and Ana Pérez-Ruiz1,2*

1 Regenerative Medicine Program, Foundation of Foundation for Applied Medical Research (FIMA), University of Navarra, Pamplona, Spain. 'Instituto de Investigación Sanitaria de Navarra (IdiSNA), Pamplona, Spain

2 Centre for Nutrition Research, Universidad de Navarra, Pamplona, Spain

3 Laboratory of Atherothrombosis, Program of Cardiovascular Diseases, FIMA Universidad de Navarra, Pamplona, CIBERCV, Spain

* Correspondence: aperez@unav.es; Tel.: +34 943394785

\begin{abstract}
Matrix metalloproteinases have been implicated in muscular dystrophy progression and recent studies described the role of MMP-10 in skeletal muscle pathology of young dystrophic mice. Nevertheless, its implication in dystrophin deficient hearts is still missing. Here, we aimed at investigating MMP-10 implication in severe muscular dystrophic progression and characterize MMP-10 loss in skeletal and cardiac muscles of aged dystrophic mice. We examined the histopathological effect of MMP-10 ablation in aged $m d x$ mice, both in the hind limb muscles and heart tissues. We have found that MMP-10 loss compromises survival rates of aged $m d x$ mice, with skeletal and cardiac muscles developing a chronic inflammatory response. Our findings indicate that MMP-10 is implicated in severe muscular dystrophy progression, identifying a new area of investigation that could lead to future therapies for dystrophic muscles.
\end{abstract}

Keywords: matrix metalloproteases, muscular dystrophy, skeletal muscle, cardiac muscle

\section{Introduction}

Duchenne muscular dystrophy (DMD) is the most devastating X-linked muscular disorder, affecting approximately 1 in 3,500 newborn males [1]. Mutations in the dystrophin gene result in a complete loss of the dystrophin protein, which links the myofiber cytoskeleton to the extracellular matrix (ECM). Consequently, dystrophic muscles are more susceptibility to muscular mechanical injury and sarcolemma damage, with myofibers undergoing continuous cycles of injury/repair. Under this chronic scenario, damage overcomes the regenerative potential of muscle, contributing to progressive muscle wasting [1]. Beyond skeletal muscle deterioration, DMD patients suffer serious heart complications, which are responsible of premature death [2]. However, cardiac implication in disease progression has not been studied as extensively as skeletal muscle pathology, and therapeutic options for DMD cardiac dysfunction are still limited.

MMPs are a family of 25 proteolytic enzymes that can degrade various components of the connective and fibrous tissues that compose the ECM, participating in its remodeling during several physiological and pathological processes [3]. Increasing evidence suggest the participation of MMPs in muscular dystrophy progression, finding gene expression and activation of various MMPs deregulated in dystrophic skeletal muscles 
from both DMD patients and animal models of muscular dystrophy [4]. Nevertheless, there is relatively little knowledge regarding the implication of MMPs in severe muscular dystrophy and considerably less information related to their role in cardiac failure associated to DMD. MMP-10 belongs to the stromelysin family of MMPs and it plays an essential role in skeletal muscle maintenance and regeneration [5-7]. In mice, healthy skeletal muscles express MMP-10, and protein levels increase in injured and dystrophic muscles [5,7]. Interestingly, genetic ablation of MMP-10 significantly impairs the deteriorated muscle phenotype of young $m d x$ mice, a mouse model of DMD, with skeletal muscles exhibiting an altered structure with delayed muscle regeneration after damage [5].

Due to the critical role that MMP-10 plays during muscular dystrophy progression and the consequences of MMPs deregulation during cardiac remodeling still [8], we aimed to better understand MMP-10 biological function in dystrophic mice. To this end, we histologically analyzed skeletal and cardiac muscles of aged $m d x$ mice to assess the implication of MMP-10 in severe muscular dystrophy.

\section{Materials and Methods}

\section{Mice}

$m d x$ and MMP-10 deficient $m d x$ animals were housed in the Animal housing facilities at the University of Navarra, according to current legislation.

\section{Tissue immunostaining and Image capture and quantification}

Skeletal muscles were frozen in isopentane cooled in liquid nitrogen. Hearts were fixed in $4 \%$ paraformaldehyde, washed in PBS, immersed into $30 \%$ sucrose/PBS and embedded in O.C.T. compound. Serial $9-\mu \mathrm{m}$ cryosections were collected at $200 \mu \mathrm{m}$ intervals through the entire tissues. Sections were blocked with $20 \%$ goat serum and immunostained with anti-eMyHC (DSHB), anti-laminin (Sigma), anti-CD45 (Biolegend) or Cy3-labeled goat anti-mouse IgG (Invitrogen). When required, fluorochrome-conjugated secondary antibodies (Molecular Probes) were applied. DAPI was used to stain nuclei. Sirius Red and H\&E stainings were performed as previously described [5].

Immunostained and stained tissue sections were viewed using a Zeiss Axiophot epi-fluorescence or a Zeiss Axio Imager M1 microscope and digital images acquired with an AxioCamMR3 or AxioCamICc3 cameras (Zeiss). Tissue section analyses, including the whole tissue transversal sections, were performed using the AxioVision and ImageJ software. Positive staining was expressed as the percentage of stained area divided by the total area of tissue. Morphometry determined the number of fibers and the cross-sectional area $(\mu \mathrm{m} 2)$ of each fiber after laminin immunostaining.

\section{Statistical analysis}

All statistical analyses were performed using SPSS 15.0 (SPSS Inc.). The Kolmogorov-Smirnov test was used to test whether measures were normally distributed. Variables were analyzed with the Mann-Whitney $\mathrm{U}$ test or Student $t$ tests. All experiments were done using at least three mice per condition. Data are expressed as means $\pm \mathrm{SEM}$. P values $<0.05$ were considered to be statistically significant.

\section{Results}

MMP-10 deficiency decreases lifespan of dystrophic mice 
In order to evaluate the implication of MMP-10 in serious muscular dystrophy, $m d x$ mice lacking MMP-10 were left to age. Compared to aged $m d x$ animals, deficiency of MMP-10 compromised animal's welfare. After noticing first signs of physical discomfort, aged MMP-10 deficient $m d x$ mice were humanely sacrificed to avoid pain and suffering (Figure 1A). On the contrary, $m d x$ mice lived longer and were sacrificed at the age of 24 months. Thus, deficiency of MMP-10 significantly decreased survival rates of dystrophic mice.
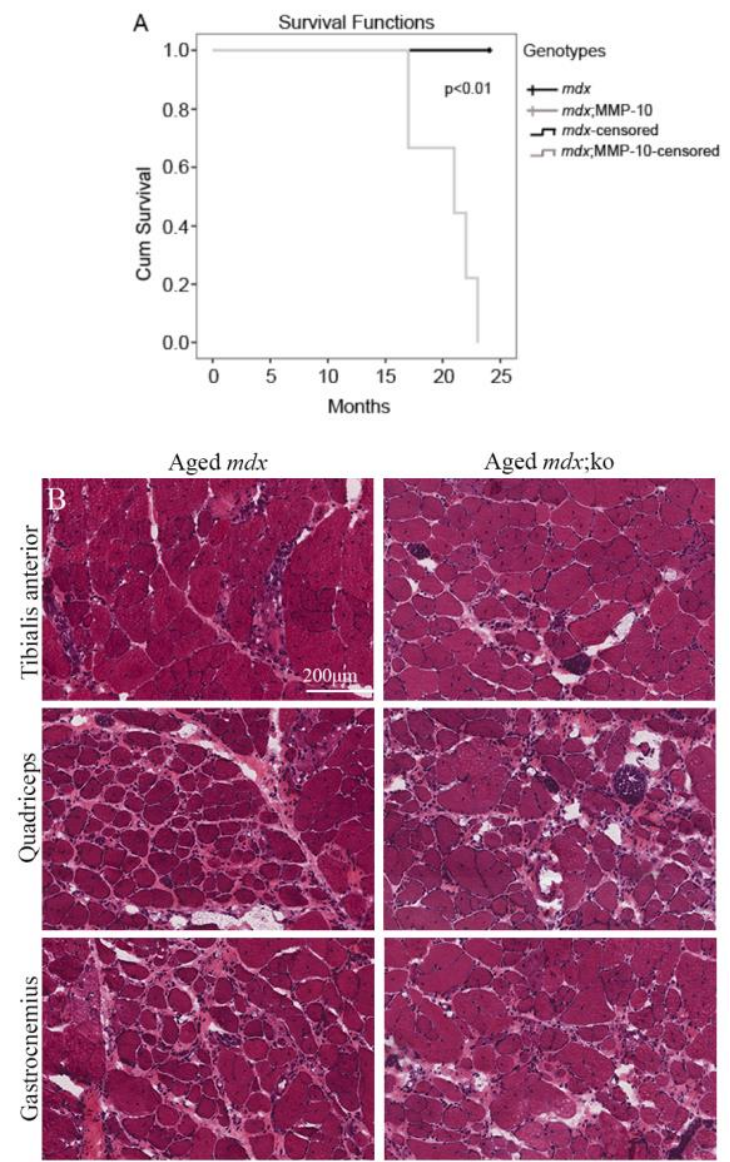

Figure 1. Loss of MMP-10 precipitates $m d x$ mice death. (A) Graph shows the survival rates between mouse genotypes. (B) Representative hematoxylin-eosin staining of tibialis anterior, quadriceps and gastrocnemius muscles isolated from aged $m d x$ and $m d x$;MMP-10 KO $(m d x ; \mathrm{ko})$ mice. Values are presented as mean \pm SEM from at least three genotypes, where * denotes a statistically significant difference from $m d x$ and $m d x$;MMP-10 KO animals $(\mathrm{P}<0.05)$.

\section{Ablation of MMP-10 deteriorates the skeletal muscle dystrophic phenotype of aged $m d x$ mice}

To assess the consequences of MMP-10 ablation in aged mdx mice, the Tibialis Anterior (TA), quadriceps and gastrocnemius muscles were isolated. Comparisons between muscles from aged $m d x$ and MMP-10 deficient $m d x$ mice showed microstructural differences. Aged MMP-10 deficient dystrophic muscles had massive muscle degeneration areas and moderate cellular infiltration, with a higher presence of necrotic fibers and fatty infiltration, in comparison to skeletal muscles from aged $m d x$ mice (Figure 1B). 
Immunostaining for CD45 confirmed the presence of infiltrating cells in muscles from aged mice, with a significant increase in the quadriceps and gastrocnemius of aged MMP-10 deficient $m d x$ mice, compared to controls (Figure 2A and C). By using a mouse IgG-Cy3 antibody we verified that loss of MMP-10 led to increased necrosis in our model, monitored by intracellular fiber staining for IgG. The TA muscles from aged MMP-10 deficient $m d x$ mice accumulated twice as many necrotic myofibers compared to aged $m d x$ animals, while quadriceps exhibited a 6-fold increase and gastrocnemius had a slightly higher necrotic areas (Figure 2B and D). Surprisingly, ablation of MMP-10 led to fibrotic accumulation only in the TAs of aged $m d x$ mice (Figure $2 \mathrm{E}$ and G). Furthermore, deficiency of MMP-10 did not impair ongoing regeneration in dystrophic muscles of aged mice, analyzed by immunostaining tissue sections for eMyHC (Figure 2F and $\mathrm{H}$ ).

Morphometric analysis after immunostaining quadriceps and gastrocnemius tissue sections for laminin showed that MMP-10 ablation did not modify the average cross sectional area of the myofibers of aged $m d x$ mice (Figure 2I-J) as well as the fiber size distribution of muscles (Figure 2I and K). Overall, our data suggest that MMP-10 deficiency aggravates the dystrophic phenotype of aged $m d x$ mice by generating a chronic inflammation response with increased fiber necrosis, without further altering muscle structure.

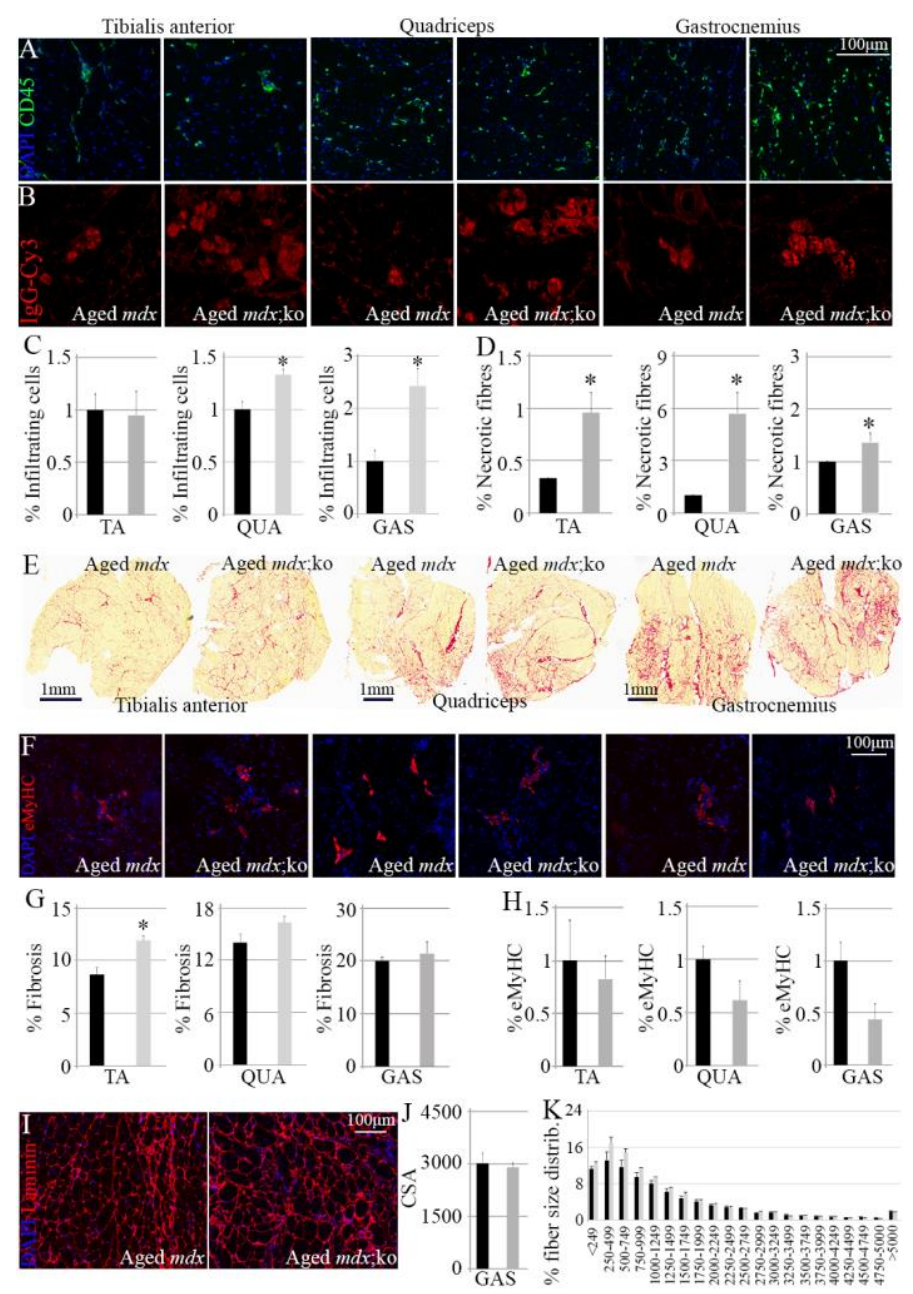

Figure 2. MMP-10 ablation deteriorates the dystrophic phenotype of aged $m d x$ mice. (A-B) Representative images of CD45 (A; quantified in C) and Cy3-IgG (B; quantified in D) immunostaining showing infiltrating cells and necrotic fibers, respectively, in muscles from $m d x$ and $m d x$;MMP-10 KO mice. (E-H) Muscle tissue sections stained for Sirius red (E) and representative images of muscles immunostained for eMyHC (F) and graphs 
showing accumulation of connective tissue $(G)$ and the presence of eMyHC positive fibers $(\mathrm{H})$ in $m d x$;MMP-10 KO mice than $m d x$ animals. (I-K) Imunostining of muscles for laminin (I) and average cross sectional area of the fibers (J) and fiber size distribution (K) in aged $m d x$ and aged MMP-10 deficient $m d x$ mice. DAPI was used to visualize nuclei. Values are presented as mean \pm SEM from at least three independent experiments. ${ }^{*}$ denotes a statistically significant difference from $m d x$ and $m d x$;MMP-10 KO animals $(\mathrm{P}<0.05)$. Abbreviations: KO, knock out.

\section{MMP-10 ablation leads to chronic inflammation in dystrophin-deficient hearts}

We next assessed changes in the hearts of aged $m d x$ mouse strains at microstructural level, finding that ablation of MMP-10 significantly increased the presence of infiltrating cells in dystrophic hearts (Figure 3A-C). However, cardiac fibers were not modified by deficiency of MMP-10, with hearts from aged mice showing similar laminin structure and expression (Figure 3A, D). Interestingly, cardiac tissues from aged MMP-10 deficient dystrophic mice accumulated less fibrotic tissue than hearts from aged $\mathrm{mdx}$ mice (Figure F-G), but these differences were not related to the fibrosis developed at ventricular left areas (Figure F, H), which usually are more affected in DMD patients.

Taken together, these findings suggest that hearts in aged $m d x ; \mathrm{MMP}-10-\mathrm{KO}$ mice exhibited a higher chronic inflammation response than aged $m d x$ mice but without further affecting cardiac structure.
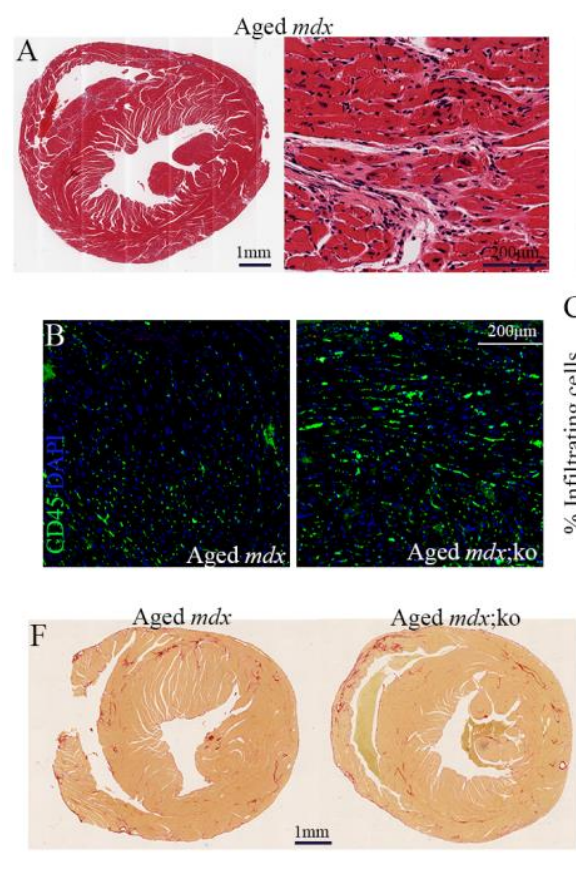
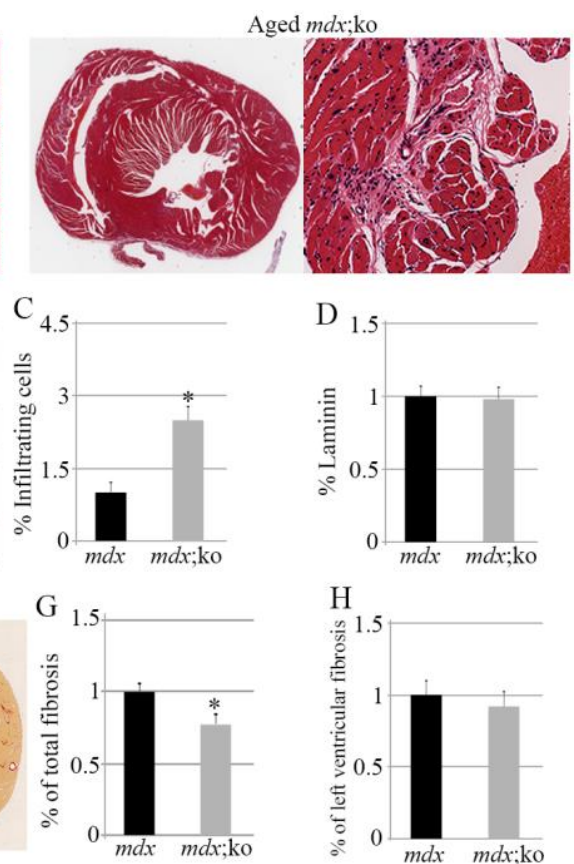

Figure 3. MMP-10 deficiency affects cardiac muscle structure in aged $m d x$ mice. (A) Representative hematoxylin-eosin staining of transversal heart sections isolated from aged $m d x$ and $m d x$;MMP-10 KO mice. Representative images of CD45 (B; quantified in C) immunostaining showing infiltrating cells in hearts from $m d x$ and $m d x$;MMP-10 KO mice. (D) Graph shows quantification of laminin expression in cardiac tissue sections. (F-H) Representative images of cardiac muscles stained for Sirius red $(\mathrm{F})$ and graphs showing fibrosis accumulation in total cardiac tissue section $(G)$ and in the ventricular left area $(H)$ in aged $m d x$ animals and aged $m d x$;MMP-10 KO mice. DAPI was used to visualize nuclei. 
Values are presented as mean \pm SEM from at three five independent experiments. ${ }^{*}$ denotes a statistically significant difference from aged $m d x$ and $m d x$; $\mathrm{MMP}-10 \mathrm{KO}$ animals $(\mathrm{P}<0.05)$. Abbreviations: KO, knock out.

\section{Discussion}

We have analyzed skeletal and cardiac muscles of aged $m d x$ mice, which reproduce more accurately human disease, to assess the implication of MMP-10 in severe muscular dystrophy. MMP-10 deficiency further to compromise skeletal muscle phenotype of aged $m d x$ mice deteriorates dystrophic hearts, with muscles developing a chronic inflammatory response that may precipitate animal death.

Although a tightly regulated, transient inflammatory response is required for normal muscle regeneration, prolonged inflammation promotes dystrophic disease pathology [9]. Increasing evidence show a complex collaborative cellular activity in muscular dystrophy progression, involving several different cell types [10]. Of these, inflammatory cells have been prominent in triggering fiber necrosis, and their effects on the overall muscle pathology in muscular dystrophy are still becoming under investigation [9]. Inflammatory cells secrete cytokines and interleukins that facilitate the myogenic functions of adult muscle stem cells in a complex balance with the activity of neutrophils, eosinophils, lymphoid cells, mast cells, macrophages and muscle resident fibro-adipogenic progenitors, influencing skeletal muscle responses during muscular disease [10-12]. Excessive activity of inflammatory cells leads to fiber necrosis, fibrosis and fatty infiltration in dystrophic muscles. In this sense, it is reasonable to think that changes in the ECM components of the muscles associated with chronic inflammation impact cell mobility and the distribution of cytokines. Therefore, ECM modulators such as MMPs may contribute to cellular instability during disease progression. According with this idea, we previously reported that genetic ablation of MMP-10 in young $m d x$ mice modified the composition of the muscle ECM and this was accompanied by increased cell infiltration and loss of muscle stem cells, worsening dystrophic phenotype [5]. Now, we support our previous findings in a context of severe muscular disease.

Although all DMD teenagers have signs of cardiac dysfunction and up to $40 \%$ of patients may die from heart complications [2], cardiac involvement has not been studied as extensively as skeletal muscle pathology, and the pathogenesis of dystrophin-deficient cardiomyopathy is not completely understood. Nevertheless, inflammatory response disrupts heart homeostasis and the degree of inflammatory cell infiltration in cardiac tissue is strongly associated with disease severity in muscular dystrophies [9], supporting our statement that MMP-10 loss led to severe dystrophic phenotype. MMPs regulate the inflammatory components of the wound healing response to diseased heart and through their direct activity on ECM components of the heart or acting as upstream signaling initiators they may initiate cellular signaling cascades [13]. Thus, ablation of MMP-10 may disrupt cellular signaling in dystrophic hearts causing serious chronic inflammation.

Our study supports ablation of MMP-10 as responsible of premature death of aged dystrophic mice. However, some considerations regarding the consequences of MMP-10 ablation in aged $m d x$ mice remain enigmatic. Differences in collagen deposits between skeletal and cardiac fibres in mutant animals and that laminin structure was not affected by MMP-10 ablation were unexpected, especially considering that these ECM components are substrates degraded by MMP-10. Recent evidence demonstrate that ablation of one MMP can be compensated by other MMP members [14]. Accordingly, activity of MMP-2 increases in MMP-10 ablated muscles [5], and MMP-2 can cleave these ECM components [3]. What is clear is that the in vivo function of the different members of the 
MMP family is extremely complex and they may cause opposing and unexpected effects on the progression of different or same diseases $[4,8,13]$, such as those affecting skeletal and cardiac muscle. Furthermore, the complexity in MMP activity increases by cell-specific induction, with some MMPs playing dual roles depending on tissue type and stage of the disease.

Future work aimed to detail the influence of MMP-10 on the inflammatory processes and its relation to deterioration of skeletal and cardiac muscles is required to understanding premature death of ablated dystrophic mice. This includes further investigation on cardiac function and its relation to the diaphragm, since loss of respiratory muscle contractility is sufficient to induce heart function failure [15]. This may open new therapeutic opportunities for DMD patients.

Author Contributions: M.B.C., M.B. and N.S. processed all the muscles and hearts for histological analyses; M.B.C. and M.B. scanned all the tissues in the automated quantitative pathology imaging system; M.B.C., M.B. and F.J.D.M. performed immunohistochemical analysis of muscle tissue; J.O. helped with the preparation of the final manuscript; A.P. designed and helped with the performance of the experiments, analyzed data and wrote the manuscript.

Funding: This work was supported by grants from the Spanish Ministry of Health [PI081919].

Institutional Review Board Statement: Institutional Review Board Statement: All the experimental procedures in rats were approved by the Directorate General of Agriculture and Livestock of the Government of Navarra and Animal Experimentation and Ethics Committee (CEEA) of the University of Navarra, following European (86/609/EEC) and Spanish (RD 53/2013) regulations for the care and use of laboratory animals.

Data Availability Statement: Data can be obtained from the corresponding authors on request.

Acknowledgments: We are grateful to the Developmental Studies Hybridoma Bank for the antibodies used in this study.

Conflicts of Interest: The authors declare no conflict of interest.

\section{References}

1. Emery AEH. The muscular dystrophies. Lancet. 2002;359(9307):687-695. doi:10.1016/S0140-6736(02)07815-7.

2. Cox G, Kunkel LM. Dystrophies and heart disease. Curr Opin Cardiol. 1997;12(3):329-343.

3. $\mathrm{Vu} \mathrm{TH}$, Werb Z. Matrix metalloproteinases: Effectors of development and normal physiology. Genes Dev. 2000;14(17):2123-2133. doi:10.1101/gad.815400

4. Ogura Y, Tajrishi MM, Sato S, Hindi SM, Kumar A. Therapeutic potential of matrix metalloproteinases in Duchenne muscular dystrophy. Front Cell Dev Biol. 2014;2(11). doi:10.3389/fcell.2014.00011

5. Bobadilla M, Sáinz N, Rodriguez J, et al. MMP-10 is required for efficient muscle regeneration in mouse models of injury and muscular dystrophy. Stem Cells. 2014;32(2):447-461. doi:10.1002/stem.1553

6. Bobadilla M, Sainz N, Abizanda G, et al. The CXCR4/SDF1 axis improves muscle regeneration through MMP-10 activity. Stem Cells Dev. 2014;23(12):1417-1427. doi:10.1089/scd.2013.0491

7. Gomez-Rodriguez V, Orbe J, Martinez-Aguilar E, et al. Functional MMP-10 is required for efficient tissue repair after experimental hind limb ischemia. FASEB J. 2014;29(3):960-972. doi:https://doi.org/10.1096/fj.14-259689

8. Soslow JH, Xu M, Slaughter JC, et al. The Role of Matrix Metalloproteinases and Tissue Inhibitors of Metalloproteinases in Duchenne Muscular Dystrophy Cardiomyopathy. J Card Fail. 2019;25(4):259-267. doi:10.1016/j.cardfail.2019.02.006

9. Nitahara-Kasahara Y, Takeda S, Okada T. Inflammatory predisposition predicts disease phenotypes in muscular dystrophy. Inflamm Regen. 2016;36(14):533-538. doi:10.1186/s41232-016-0019-0

10. Morgan J, Partridge T. Skeletal muscle in health and disease. DMM Dis Model Mech. 2020;13(2):15-17. doi:10.1242/dmm.042192

11. Tidball JG, Welc SS, Wehling-Henricks M. Immunobiology of Inherited Muscular Dystrophies. Compr Physiol. 2018;8(4):1313-1356. doi:10.1002/cphy.c170052

12. Joe AWB, Yi L, Natarajan A, et al. Muscle injury activates resident fibro/adipogenic progenitors that facilitate myogenesis. Nat Cell Biol. 2010;12(2):153-163. doi:10.1038/ncb2015

13. Lindsey ML, Iyer RP, Jung M, DeLeon-Pennell KY, Ma Y. Matrix metalloproteinases as input and output signals for post-myocardial infarction remodeling. J Mol Cell Cardiol. 2016;91:134-140. doi:10.1016/j.yjmcc.2015.12.018 
14. Ra HJ, Parks WC. Control of matrix metalloproteinase catalytic activity. Matrix Biol. 2007;26(8):587-596. doi:10.1016/j.matbio.2007.07.001

15. Melacini P, Vianello A, Villanova C, et al. Cardiac and respiratory involvement in advanced stage duchenne muscular dystrophy. Neuromuscul Disord. 1996;6(5):367-376. doi:10.1016/0960-8966(96)00357-4 